Identification of Homo-Oligomers as Potential Intermediates in Acetylcholine Receptor Subunit Assembly

David J. Anderson, and Gunter Blobel

PNAS 1983;80;4359-4363

doi:10.1073/pnas.80.14.4359

This information is current as of December 2006.

\begin{tabular}{|ll|}
\hline & $\begin{array}{l}\text { This article has been cited by other articles: } \\
\text { www.pnas.org\#otherarticles }\end{array}$ \\
E-mail Alerts & $\begin{array}{l}\text { Receive free email alerts when new articles cite this article - sign up in the box at the top } \\
\text { right corner of the article or click here. }\end{array}$ \\
Rights \& Permissions & $\begin{array}{l}\text { To reproduce this article in part (figures, tables) or in entirety, see: } \\
\text { www.pnas.org/misc/rightperm.shtml }\end{array}$ \\
Reprints & $\begin{array}{l}\text { To order reprints, see: } \\
\text { www.pnas.org/misc/reprints.shtml }\end{array}$ \\
\hline
\end{tabular}

Notes: 


\title{
Identification of homo-oligomers as potential intermediates in acetylcholine receptor subunit assembly
}

(multi-subunit membrane protein/detergent solubilization/sucrose gradient analysis/homologous associations/early biosynthetic events)

\author{
David J. Anderson* and Günter Blobel \\ Laboratory of Cell Biology, The Rockefeller University, New York, New York 10021 \\ Communicated by Philip Siekevitz, April 4, 1983
}

\begin{abstract}
We have examined the sedimentation behavior, on sucrose density gradients, of acetylcholine receptor (AcChoR) subunits synthesized in vitro and integrated into heterologous rough microsomal membranes. In media containing nondenaturing detergents such as Triton X-100 or deoxycholate, the subunits appear to self-associate although, as previously reported, no heterologous interactions were detected. The sedimentation profiles assume a broad distribution in the region of 7-13 S. However, the peak fractions occupy the same region of the gradient as does native AcChoR, run in parallel. Such large homo-oligomers were not observed for another membrane protein, opsin, studied in the same way. This indicated that the associations are indeed between the AcChoR subunits and not simply between all newly synthesized membrane proteins. The homologous associations are interpreted to suggest a mechanism for maintaining the ionophore surfaces of the subunits in an energetically preferred, but metastable, configuration during the lengthy period of post-translational assembly.
\end{abstract}

The oligomeric assembly of the acetylcholine receptor (AcChoR) subunits poses a unique problem in membrane protein biogenesis-that is, how four distinct transmembrane glycoproteins, translated from separate mRNAs (1), are assembled into a quaternary complex that forms a hydrophilic ion channel through the lipid bilayer. The problem is further complicated by the observation that AcChoR subunit assembly does not occur immediately upon insertion of the subunits into the rough endoplasmic reticulum (RER) membrane (1) but rather after a delay of at least $30 \mathrm{~min}$, or perhaps even longer (2). This fact implies that $(i)$ newly synthesized AcChoR subunits must be transported to a common subcellular site, perhaps the Golgi apparatus $(2,3)$, for assembly to occur, and (ii) the individual subunits must assume a stable configuration in the lipid bilayer of intracellular organelles, for a considerable time prior to formation of the ionophore complex.

The AcChoR subunits are comprised by four polypeptides$\alpha, \beta, \gamma$, and $\delta$, of apparent molecular masses of $40,50,60$, and 65 kilodaltons $(\mathrm{kDa})$, respectively $(4)$. Their stoichiometry in the mature complex is $\alpha_{2} \beta \gamma \delta$ (5-7). These subunits are synthesized with signal sequences $(8-10)$ and are cotranslationally integrated into the RER membrane (1) by a mechanism that utilizes the recently purified $(11,12)$ signal recognition particle (8). Although these subunits are not associated with one another at this stage in their biosynthesis (1), they exhibit a transmembrane topology that is similar, at least in its gross aspects, to that observed for their counterparts in the mature hetero-oligomer $(13,14)$.

In this study we have examined the sedimentation behavior of these early biosynthetic forms of the AcChoR subunits on

The publication costs of this article were defrayed in part by page charge payment. This article must therefore be hereby marked "advertisement" in accordance with 18 U.S.C. $\$ 1734$ solely to indicate this fact. sucrose density gradients. The subunits each appear to self-associate, forming homo-oligomers in the range of 7-13 S. Such homologous associations were not observed for another membrane protein, opsin, studied in an identical system in parallel. These AcChoR homo-oligomers are suggested to function in the stabilization of hydrophilic surfaces that will eventually border the ion channel in the mature complex, during the lengthy period of transport to the site of assembly of the functional receptor.

\section{MATERIALS AND METHODS}

In vitro synthesis of AcChoR subunits and of opsin were carried out as described $(1,15)$. Solubilization of total translation reaction mixtures was carried out by adjusting the KOAc to 0.3 $\mathrm{M}$ and then adding $1 / 19$ th vol of $20 \%$ Triton X-100, followed by incubation on ice for $30 \mathrm{~min}$. Prior to sucrose gradient analysis, insoluble material was removed by centrifugation at 100,000 $\times g_{a v}$ for $10 \mathrm{~min}$ in an airfuge (Beckman). Velocity sedimentation analysis was performed essentially after the method of Martin and Ames (16) on 5-ml linear gradients consisting of 10 $40 \%$ (wt/wt) sucrose, $50 \mathrm{mM}$ Tris $\cdot \mathrm{HCl}(\mathrm{pH} 7.4), 0.1 \mathrm{M} \mathrm{KOAc}$, $10 \mu \mathrm{g}$ of gelatin per $\mathrm{ml}(17), 0.1 \%$ Trasylol, and $0.1 \%$ Triton $\mathrm{X}$ 100 (except where indicated otherwise). Ten fractions of $\approx 0.5$ $\mathrm{ml}$ each were collected, by time, from each gradient; it was impractical to collect more fractions as each fraction had to be analyzed by immunoprecipitation and $\mathrm{NaDodSO}_{4} /$ polyacrylamide gel electrophoresis. For immunoprecipitation from the gradient fractions under nondenaturing conditions, each fraction was made $1.0 \%$ in Triton $\mathrm{X}-100$, and incubation with antibody (5-10 $\mu \mathrm{l}$ per fraction) was for $12 \mathrm{hr}$ at $4^{\circ} \mathrm{C}$. Antibody-antigen complexes were adsorbed to protein A-Sepharose (Pharmacia) and washed four times as described (1) prior to solubilization in $\mathrm{NaDodSO}_{4}$ /polyacrylamide gel electrophoresis sample buffer. Electrophoresis was performed throughout on $12 \%$ polyacrylamide gels, except for opsin, which was analyzed on $15 \%$ polyacrylamide/8 M urea gels as described (15). Quantitation was accomplished by scanning gel lanes on the preflashed (18) $x$-ray films and integrating peak areas by using an automatic densitometer (Beckman). Values of $s_{20, w}$ were calculated by the method of McEwen (19) by using interpolated values of \% sucrose at the peaks and time integral values provided in tables.

For analysis of native receptor (nAcChoR), receptor-enriched membranes from Torpedo were prepared as described (20) and peripheral membrane polypeptides were removed by $\mathrm{pH} 11$ extraction (21).

Abbreviations: AcChoR, acetylcholine receptor; nAcChoR, native AcChoR; kDa, kilodalton(s); RER, rough endoplasmic reticulum.

* Present address: Dept. of Biochemistry, Institute of Cancer Research, Columbia Univ. College of Physicians and Surgeons, $701 \mathrm{~W}$. 168th St., New York, NY 10032. 


\section{RESULTS}

For our studies of early biosynthetic forms of the AcChoR polypeptides, we have synthesized the subunits by in vitro translation of Torpedo californica total mRNA in a wheat germ cellfree system, supplemented with canine pancreas microsomal membranes. As each of the AcChoR subunits synthesized in vitro represents ca. $0.5 \%$ of the total translation products (1), the presence of these $\left[{ }^{35} \mathrm{~S}\right]$ methionine-labeled subunits in fractions from a sucrose density gradient could only be ascertained by immunoprecipitation with subunit-specific antibodies (1), followed by electrophoresis and fluorography (22). These antibodies were raised against AcChoR subunits eluted from preparative $\mathrm{NaDodSO}_{4}$ /polyacrylamide gels, and immunoprecipitation of authentic AcChoR denatured in $\mathrm{NaDodSO}_{4}$ (which dissociates the constituent polypeptides) is therefore subunitspecific (Fig. 1, "NaDodSO 4 immunoprecipitates," lanes 1-4). However, when immunoprecipitation is performed in the presence of a nondenaturing detergent such as Triton X-100, all four subunits are coprecipitated by each antibody (Fig. 1, "Triton X-100 immunoprecipitates," lanes 1-4). Thus, our antisera are capable of detecting heterologous subunit associations, under nondenaturing conditions.

The $\delta(65 \mathrm{kDa})$ subunit was chosen for the initial sedimentation analysis, as we had previously characterized this chain in some detail $(1,8)$. On $10-40 \%$ sucrose gradients containing 0.1 M KOAc and 0.1\% Triton X-100, AcChoR- $\delta$ assumed a broad but symmetrical distribution centering around $9.5 \mathrm{~S}$ [Fig. $2 \mathrm{~A}$ (closed circles) and $B$ ] and extending from 7 to $13 \mathrm{~S}$. [All $s$ values have been calculated according to McEwen (19) and were consistent with the location of $\mathrm{nAcChoR}$ monomer and dimer, indicated by arrows in Fig. 2A]. The same distribution was obtained when the microsomes were reisolated and solubilized at a 4-fold greater dilution [Fig. $2 \mathrm{~A}$ (open triangles) and $C$ ]. The peak fractions of AcChoR- $\delta$ comigrated with authentic AcChoR run in parallel [Fig. $2 A$ (arrows, fractions 4 and 5 ), $B, C$, and $E]$, although the in vitro-synthesized material was more heterogeneous than its authentic counterpart. Interestingly, preAcChoR- $\delta$, synthesized in the absence of rough microsomes (l) and analyzed in the absence of detergent, also migrated as a larger than monomeric species (Fig. 2A, open circles; compare

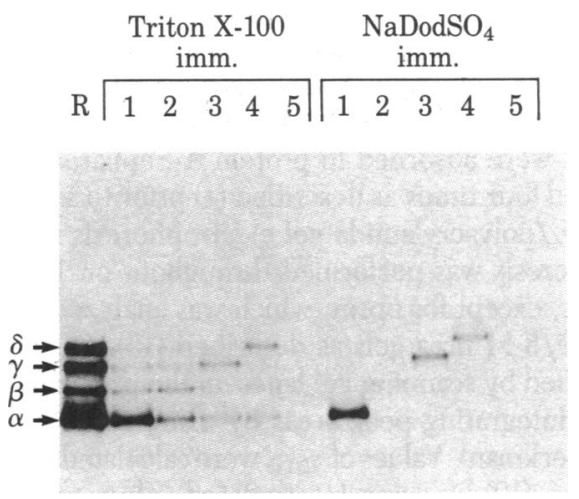

FIG. 1. Characterization of anti-AcChoR subunit antisera under denaturing versus nondenaturing conditions. Triton X-100 immunoprecipitates (imm.): immunoprecipitation was performed after dilution of $9 \mathrm{~S}$, ${ }^{225}$-labeled nAcChoR (1) into immunoprecipitation buffer containing $1 \%$ Triton X-100 and $0.2 \% \mathrm{NaDodSO}_{4} \cdot \mathrm{NaDodSO}_{4}$ immunoprecipitates (imm.): immunoprecipitation was performed after prior denaturation of the ${ }^{125} \mathrm{I}$-labeled AcChoR in $1 \% \mathrm{NaDodSO}_{4}$. Antisera used in lanes $1-5$ were anti- $\alpha,-\beta,-\gamma,-\delta$, and nonimmune, respectively. The nonstoichiometric recovery of the four subunits in each of the Triton X-100 immunoprecipitates is most likely due.to partial dissociation of the AcChoR complex arising from the iodination procedure (1). $R$, AcChoR.
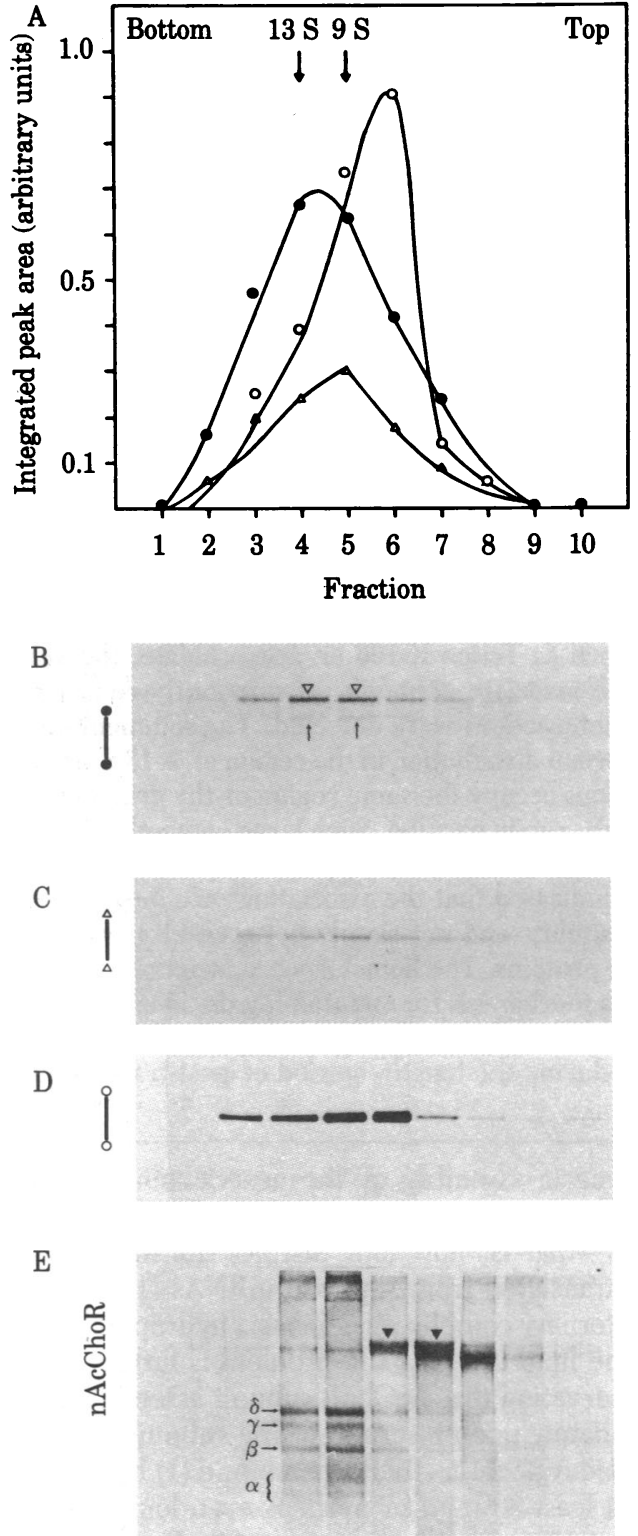

FTG. 2. Sucrose gradient analysis of in vitro-synthesized AcChoR$\delta$. (A) Quantitative representation of the gel profiles shown in $B-D$, obtained by a densitometric scan of the autoradiographic films. The band quantitated by this procedure is indicated (in all ensuing figures as well) by an open arrowhead, wherever multiple forms appear on the gel. [ $A$ (Closed circles) and $B]$ AcChoR- $\delta$, obtained by analysis of total reaction mixture. Upward arrows in $B$ indicate the nonglycosylated and partially glycosylated forms characterized previously; open arrowheads indicate the fully glycosylated form (8). [A (Open triangles) and $C$ ] AcChoR- $\delta$, solubilized from reisolated microsomes at a 4-fold greater dilution than that used in $B$. [A (Open circles) and $D$ ] pre-AcChoR- $\delta$, synthesized in the absence of microsomal membranes, analyzed on gradients not containing detergent. $(E)$ AcChoR-enriched Torpedo membranes solubilized in 1\% Triton X-100/0.3 M KOAc and analyzed in parallel with the samples in $B-D$. The gradient fractions were precipitated with trichloroacetic acid for analysis of the total protein components; shown is the Coomassie blue-stained gel. The four AcChoR subunits are indicated by horizontal arrows; the $\alpha$ chain runs as a smeared doublet due to heating and trichloroacetic acid precipitation. Arrowheads indicate the putative large subunit of the $\mathrm{Na}^{+}, \mathrm{K}^{+}$-ATPase. Arrows in $A$ indicate the peak fractions (4 and 5) containing nAcChoR (see $E$ ). Fraction 4 is calculated (19) to be $12 \mathrm{~S}$, whereas fraction 5 is $9 \mathrm{~S}$. Although we have assumed (arrows) that fraction 4 contains some 13S AcChoR, the resolution of our fractionation is not sufficient for us to determine the actual amount of dimeric receptor. Each fraction spans ca. $3 \mathrm{~S}$ units. 
Fig. $2 B$ and $D$ ). [The lower $s$ value for pre-AcChoR- $\delta$ is most likely due to the absence of the three core oligosaccharide groups (8), which contribute ca. $5 \mathrm{kDa}$ of mass.] Most important, the electrophoretic profile of the anti- $\delta$-immunoprecipitated gradient fractions (Fig. $2 B$ and $C$ ) indicated that no subunits other than AcChoR- $\delta$ had been brought down under these nondenaturing conditions, so that the relatively high $s$ values must be due to homologous associations between AcChoR- $\delta$ chains. Only the $35-$ to $75-\mathrm{kDa}$ region of the gel is shown; the remainder contained no other bands.

It could be argued that the observed high $s$ values were due to associations between AcChoR- $\delta$ and some (unlabeled) component of the endoplasmic reticulum translocation machinery (17). Such an association would be expected to occur for any membrane protein synthesized in vitro. To test this, we examined the opsin protein of retinal rod outer segments. When solubilized from its native disk membranes by Triton X-100, opsin migrates as a $4.3 \mathrm{~S}$ species (23) in sucrose density gradients. When in vitro-synthesized and membrane-integrated (15) opsin was solubilized in 1.0\% Triton X-100/0.3 M KOAc and analyzed exactly as in the case of AcChoR- $\delta$, it migrated as a symmetrical, $4.3 \mathrm{~S}$ species [Fig. $3 \mathrm{~A}$ (open circles) and $\mathrm{B}$ ] with a much narrower distribution than that seen for AcChoR$\delta$ [compare Figs. 2 and $3 A$ (closed circles) and $C$ ]. This result indicated that the sedimentation profile of AcChoR- $\delta$ could not be explained by invoking aggregation with constitutive proteins contained in the rough microsomal membranes.

To further investigate the stability of the AcChoR- $\delta$ homo-

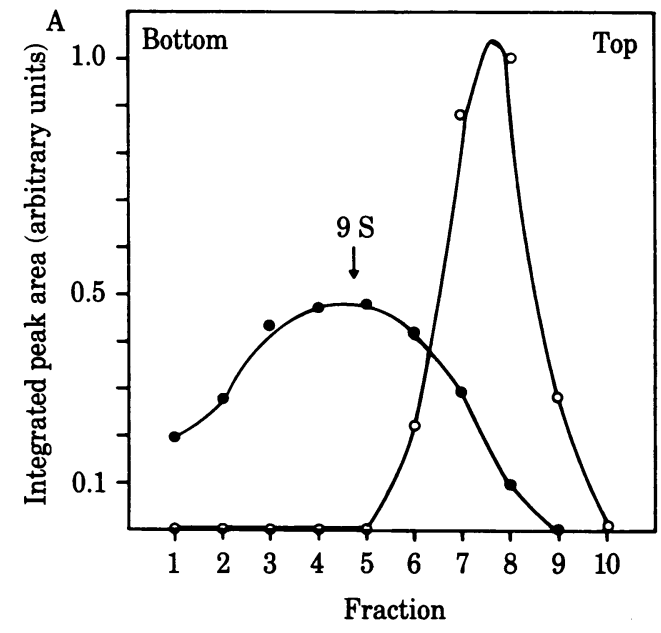

B

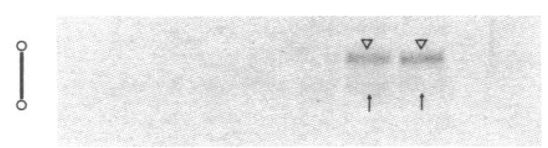

C

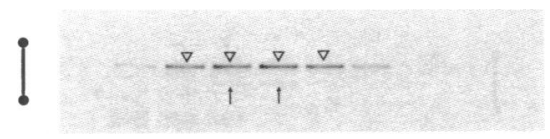

FIG. 3. Comparative sedimentation analysis of AcChoR- $\delta$ and op$\sin$. (A) Sedimentation profiles reconstructed from densitometric scans of the gels shown in $B$ and $C$. [ $A$ (Open circles) and $B$ ] opsin. Arrowheads indicate the core-glycosylated, membrane-integrated form of opsin (15); upward arrows indicate the residual unintegrated precursor (or integrated, nonglycosylated material), which smears slightly into the gradient. [ $A$ (Closed circles) and $C$ ] AcChoR- $\delta$ analyzed in parallel. Arrowheads indicate the fully glycosylated form; upward arrows indicate the residual precursor. AcChoR- $\delta$ in this experiment assumed a slightly broader distribution than on other occasions (see Figs. 2, 4, and 5).
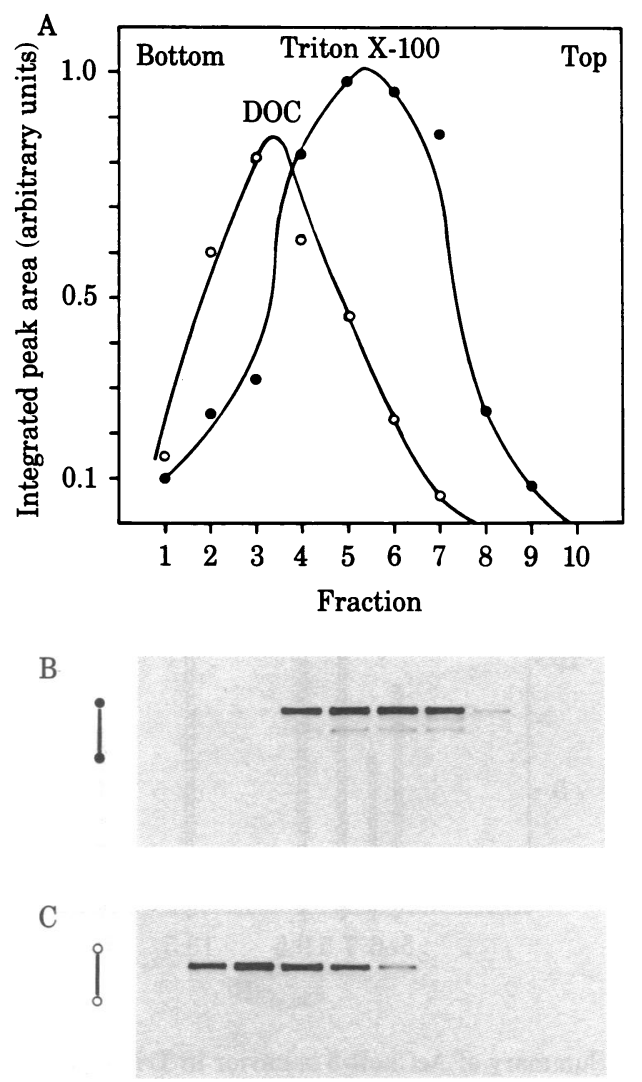

FTG. 4. Comparative sedimentation analysis of AcChoR- $\delta$ in Triton X-100 and deoxycholate (DOC). [A (Closed circles) and B] AcChoR$\delta$ after solubilization in $1 \%$ Triton X-100/0.3 M KOAc exactly as indicated previously (Figs. 2 and 3). [A (Open circles) and C] AcChoR- $\delta$ in DOC. Rough microsomes containing AcChoR- $\delta$ were reisolated from in vitro translation reaction mixtures and diluted 4-fold into $0.05 \mathrm{M}$ Tris $\cdot \mathrm{HCl}, \mathrm{pH} 8.0 / 0.1 \mathrm{M} \mathrm{NaCl} / 0.5 \% \mathrm{Na}$ DOC. The sample was analyzed on a $10-40 \%$ sucrose gradient containing this same buffer, except with $0.1 \% \mathrm{DOC}$; prior to immunoprecipitation the fractions were adjusted to $1 \%$ Triton X-100.

oligomers, we examined the behavior of this subunit in deoxycholate. This detergent, although nondenaturing, is considered to be harsher than Triton X-100 (24). Deoxycholate-solubilized AcChoR- $\delta$, in $0.05 \mathrm{M}$ Tris $\cdot \mathrm{HCl}, \mathrm{pH} 8.0 / 0.1 \mathrm{M} \mathrm{NaCl}$, also migrated as an oligomeric species [Fig. $4 \mathrm{~A}$ (open circles) and $C$ ]. However, it sedimented faster (peak at $16 \mathrm{~S}$ ) than did AcChoR- $\delta$ in Triton X-100 [compare Fig. 4 A (closed circles) and $B]$. This is to be expected (25), as the partial specific volume of deoxycholate is lower than that of Triton X-100.

The sedimentation behavior of AcChoR- $\delta$ in $0.1 \%$ Triton X100 , averaged over three separate experiments, is summarized in Fig. 5. Over $75 \%$ of the material distributed between $7.5 \mathrm{~S}$ and 13.5 S. This distribution was not affected by raising the level of Triton X-100 in the gradient to $1.0 \%$ or by raising the salt level to $0.3 \mathrm{M}$ (data not shown). The oligomers were also maintained in the presence of the detergent octyl- $\beta$-D-glucoside (not shown). These measurements are not sufficiently precise (6) to allow calculations of the number of molecules contained in the homo-oligomers. Moreover, such calculations require values for detergent binding, and the measurement of such binding (26) is not compatible with the radiochemical quantities of material synthesized in vitro. Nonetheless, under a variety of conditions of ionic strength, $\mathrm{pH}$, and type of detergent, AcChoR- $\delta$ sediments as a greater than monomeric species, to about the same position as does nAcChoR, and this profile is not seen for an unrelated membrane protein, opsin. 


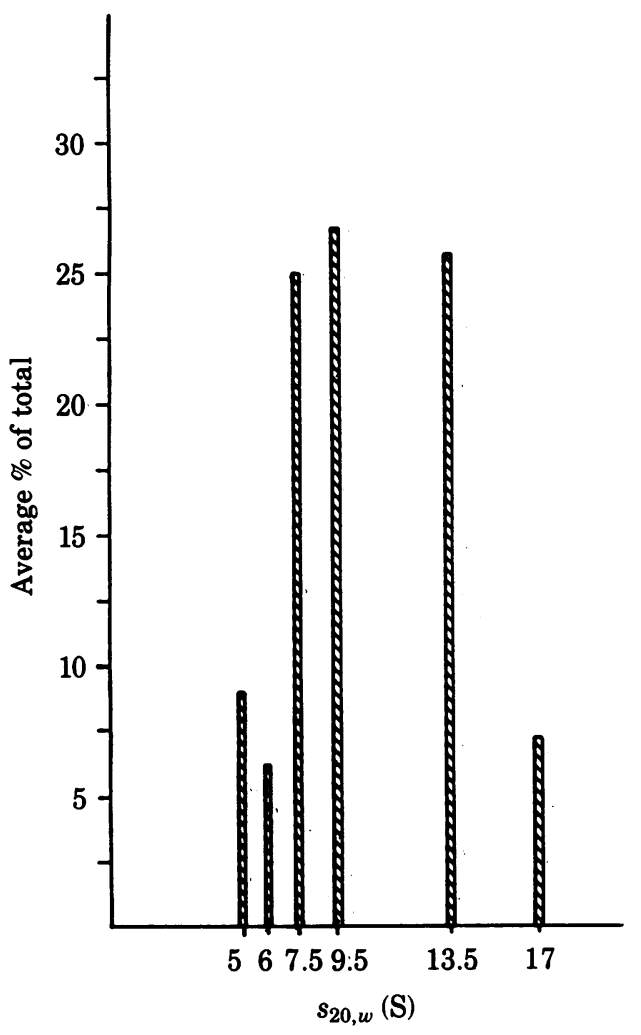

Fig. 5. Summary of AcChoR- $\delta$ behavior in Triton X-100-containing sucrose gradients. The material in the peak fractions of three different AcChoR- $\delta$ gradients (Figs. 2-4) was expressed as \% of the total material in the gradient. (A peak was defined as a fraction containing at least $18 \%$ of the total; the highest peaks contained $22 \%$ of the total Each gradient contained three or four peak fractions.) These \% of total values, calculated for each of the $s_{20, w}$ s indicated on the horizontal axis, were then summed from the three experiments and normalized to $100 \%$. These values are indicated on the vertical axis.

The other three AcChoR subunits exhibited homologous associations as well (Figs. 6 and 7). As in the case of AcChoR- $\delta$, electrophoretic analysis of the immunoprecipitated gradient fractions (Figs. $6 B$ and $7 B$ and $C$ ) indicated that the subunit associations were exclusively homologous, even though all four subunits were present in every sample. [The small amounts of $\delta$ seen in the $\beta$ and $\gamma$ samples is due to antibody crossreactivity, even after $\mathrm{NaDodSO}_{4}$ denaturation, as previously documented (1).] That the distribution of AcChoR- $\beta$ appears asymmetric (Fig. $7 A$ ) is due to the low intensity of the gel bands (Fig. 7B). (Multiple scans of such faint bands gave values varying by as much as 20\%; in such cases the gel itself is more informative than the graph.) The $\alpha$ peak centered around $9 \mathrm{~S}$ (Fig. 6A), whereas $\gamma$ and $\beta$ migrated around $13 \mathrm{~S}$ and 7-8. S, respectively (Fig. 7A). In Fig. $7 \boldsymbol{B}$, the $\delta$ subunit peak (closed arrowheads) is displayed by one fraction from the $\boldsymbol{\beta}$ peak (open arrowheads). This demonstrates that the separation between the subunits occurred during sedimentation and not during the subsequent immunoprecipitation steps.

\section{DISCUSSION}

We have examined the sedimentation behavior of AcChoR subunits at a stage in their biosynthesis when they have been asymmetrically integrated into the RER membrane but have not yet assembled into a functional receptor-ionophore complex. Our results indicate that each subunit forms homo-oligomers, heterogeneous in size but averaging in the region of the nAcChoR. (Rough calculations suggest the oligomers might consist of four to eight monomers.) In the case of the $\delta$ subunit, these large

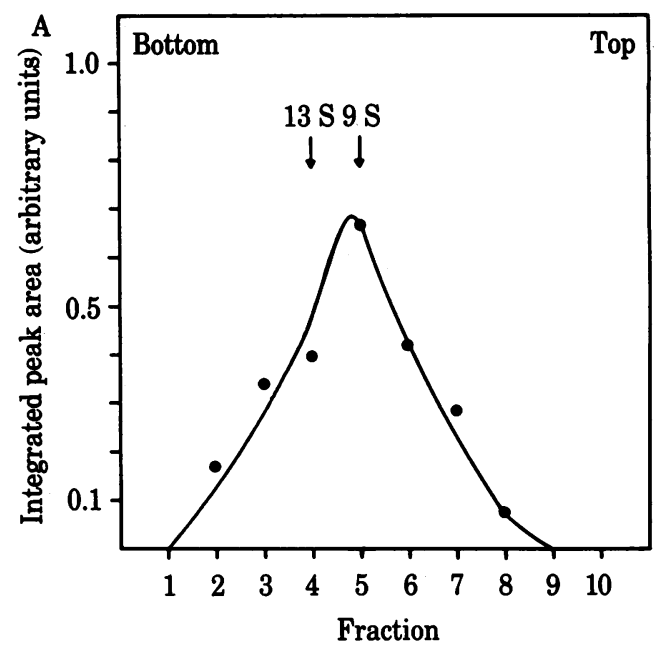

B

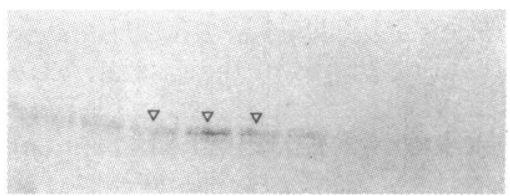

Fig. 6. Sucrose gradient analysis of AcChoR- $\alpha$. The experiment was performed exactly as in Figs. 2 and 3, except that anti- $\alpha$ antibody was used to immunoprecipitate each gradient fraction.

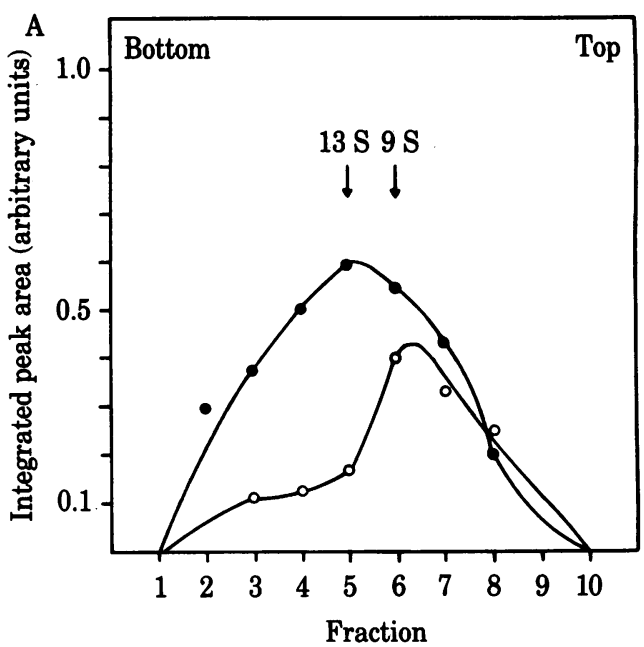

$\mathrm{B}$

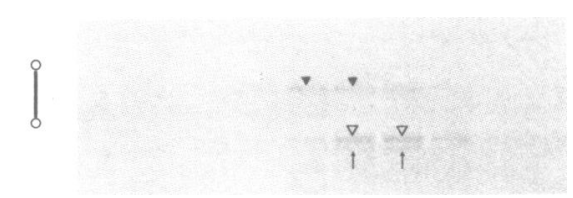

C

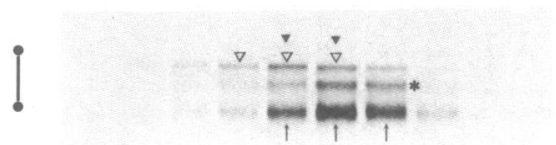

Fig. 7. Sucrose gradient analysis of AcChoR- $\beta$ and AcChoR- $\gamma$. [A (Open circles) and $B$ ] AcChoR- $\beta$. Open arrowheads $(B)$ indicate glycosylated form of $\beta$; upward arrows indicate the membrane-integrated but nonglycosylated form (1). Filled arrowheads indicate AcChoR- $\delta$, which appears due to antibody crossreactivity manifested when excess amounts of antiserum are used (1). [A (Closed circles) and C] AcChoR$\gamma$. Open arrowheads indicate fully glycosylated form of AcChoR- $\gamma(8)$; the asterisk indicates a partially glycosylated form; and upward arrows indicate residual unprocessed pre-AcChoR- $\gamma(1,8)$. Closed arrowheads indicate AcChoR- 8 present due to crossreactivity (1). 
complexes were seen in several different types of detergents and were not dissociated by changes in salt, $\mathrm{pH}$, or divalent cations. Furthermore, pre-AcChoR- $\delta$ self-associated in the absence of any detergent (Fig. 2D). All four AcChoR subunits formed homo-oligomers, but such large molecular forms were not exhibited by opsin, an integral membrane protein of comparable molecular mass, when analyzed after a similar in vitro synthesis. As opsin shares the cotranslational integration process (15) in common with the AcChoR subunits $(1,8)$, the high $s$ values seen for the AcChoR subunits are unlikely to be due to an association with nonradioactive components of the RER translocation machinery $(11,17)$, as opsin would in that case exhibit a similar sedimentation profile. However, we cannot rigorously rule out the possibility that the AcChoR subunits all aggregate "nonspecifically" with some unlabeled RER proteins, which do not interact with opsin.

It is a reasonable hypothesis, based on the appearance of the negatively stained receptor $(27,28)$, that in the mature AcChoR complex, each of the subunits borders the ion channel. By analogy to the case of the well-characterized alamethicin channel (29), the ionophore boundary surfaces of the subunits are likely to contain polar (or charged) regions of the protein helix. One rationalization of the homo-oligomers documented here is that these structures are an energetically preferred configuration, which maximizes contact between the polar ionophore surfaces and minimizes their interaction with the hydrophobic interior of the lipid bilayer. Such a configuration would, in effect, mimic that of the mature AcChoR complex with the exception that it need not be of a precise stoichiometry. As multiple copies of each subunit are being synthesized by the ribosomes on its corresponding mRNA, these associations could form either during, or immediately after, synthesis, when the local concentration of any given subunit in the RER would be extremely high. On the other hand, the different subunits would be extremely dilute with respect to one another as they are translated from separate mRNAs (1). Thus, spatial considerations favor homologous over heterologous associations, early in AcChoR biogenesis. Finally, homo-oligomer formation by the AcChoR subunits is attractive for evolutionary reasons, irrespective of thermodynamic considerations. Sequence analysis (5) has indicated that the four subunits arose by a process of gene duplication and divergence. Thus, "ancestral" AcChoRs may have been homooligomers, as is the contemporary gap junction channel (30), so that there would be evolutionary "precedent" for the complexes observed here.

A major problem in the interpretation of our observations is the inability to extend them to an in vivo system, in which early biosynthetic forms of all four subunits can be studied. On this basis the physiological relevance of our data can be questioned. Unfortunately, routine primary culture of Torpedo electrocytes is currently not feasible. Merlie and Sebbane (2) have been able to study synthesis of the $\alpha$ and $\beta$ subunits of the muscle AcChoR in a tumor cell line, $\mathrm{BC} 3 \mathrm{H}-1$. However, they were unable to detect synthesis of the other chains after short pulses of label.

If in fact the AcChoR subunits are initially self-associated, then subunit-subunit interchange between the different homooligomers must occur at some point in biogenesis. In that case, the heterologous associations would have to be of higher af- finity than the homologous ones; the broad sedimentation profile of the homo-oligomers is consistent with the constraint that they be held together by relatively low-affinity interactions. Furthermore, the subunit exchanges might be rendered irreversible by the glycosylation reactions known to occur in the Golgi apparatus. It is also possible that heterologous assembly is a facilitated process, involving components (21) extrinsic to the actual AcChoR complex.

We are grateful to Drs. Reid Gilmore, Arthur Karlin, and Ari Helenius for helpful discussions during the course of these experiments. This work was supported by a grant from the Muscular Dystrophy Foundation.

1. Anderson, D. J. \& Blobel, G. (1981) Proc. Natl. Acad. Sci. USA 78, 5598-5602

2. Merlie, J. P. \& Sebbane, R. (1981) J. Biol. Chem. 256, 3605-3608.

3. Fambrough, D. M. \& Devreotes, P. N. (1978) J. Cell Biol 76, 237244

4. Weill, C. L., McNamee, H. G. \& Karlin, A. (1974) Biochem. Biophys. Res. Commun. 61, 997-1003.

5. Raftery, M. A., Hunkapiller, M. W., Strader, C. B. D. \& Hood, L. E. (1980) Science 208, 1454-1457.

6. Reynolds, J. \& Karlin, A. (1978) Biochemistry 17, 2035-2038.

7. Lindstrom, J., Merlie, J. \& Yogeeswaran, G. (1979) Biochemistry 18, 4465-4469.

8. Anderson, D. J., Walter, P. \& Blobel, G. (1982) J. Cell Biol. 93, 501-506.

9. Claudio, T., Ballivet, M., Patrick, J. \& Heinemann, S. (1983) Proc. Natl. Acad. Sci. USA 80, 1111-1115.

10. Noda, M., Takahashi, H., Tanabe, T., Toyosato, M., Furutani, Y., Hirose, T., Asai, M., Inayama, S., Miyata, T. \& Numa, S. (1982) Nature (London) 299, 793-797.

11. Walter, P. \& Blobel, G. (1982) Nature (London) 299, 691-698

12. Walter, P. \& Blobel, G. (1980) Proc. Nath Acad. Sci. USA 77, 71127116.

13. Anderson, D. J., Tzartos, S. J., Gullick, W., Lindstrom, J. \& Blobel, G. (1983) J. Neurosci., in press.

14. Wennogle, L. P., Oswald, R., Saitoh, T. \& Changeux, J.-P. (1981) Biochemistry 220, 2492-2497.

15. Goldman, B. M. \& Blobel, G. (1981) J. Cell Biol. 90, 236-242.

16. Martin, R. G. \& Ames, B. N. (1961) J. Biol. Chem. 236, 1372-1379.

17. Gilmore, R., Walter, P. \& Blobel, G. (1982) J. Cell Biol. 95, 470477.

18. Laskey, R. A. \& Mills, A. D. (1975) Eur. J. Biochem. 56, 335-341.

19. McEwen, C. R. (1967) Anal Biochem. 20, 114-149.

20. Klymkowsky, M. W., Heuser, J. E. \& Stroud, R. M. (1980) J. Cell Biol 85, 823-838.

21. Neubig, R. R., Krodel, E. R. Boyd, N. D. \& Cohen, J. B. (1979) Proc. Natl. Acad. Sci. USA 76, 690-694.

22. Bonner, W. M. \& Laskey, R. A. (1974) Eur. J. Biochem. 46, 8388 .

23. Clarke, S. (1975) J. Biol. Chem. 250, 5459-5469.

24. Helenius, A., Fries, E., Garoff, M. \& Simons, K. (1976) Biochim. Biophys. Acta 436, 319-334.

25. Helenius, A. \& Simons, K. (1975) Biochim. Biophys. Acta 445, 229 279.

26. Wise, D. S., Karlin, A. \& Schoenborn, B. P. (1979) J. Mol. Biol. 28, 473-496.

27. Wise, D. S., Wall, J. \& Karlin, A. (1981) J. Biol Chem. 256, 1262412627.

28. Kistler, J., Stroud, R. M., Klymkowsky, M. W., Lalancette, R. A. \& Fairclough, R. H. (1982) Biophys. J. 37, 371-383.

29. Fox, R. O. \& Richards, F. M. (1982) Nature (London) 300, 325330 .

30. Hertzberg, E. L. \& Gilula, N. B. (1979) J. Biol. Chem. 254, 21382147. 